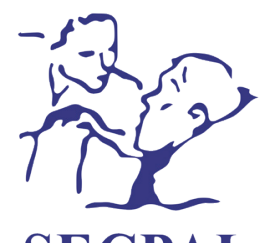

Medicina Paliativa

www. medicinapaliativa.es

ORIGINAL

\title{
Versión española del Hospice Comfort Questionnaire para evaluar el confort en pacientes en cuidados paliativos: adaptación transcultural y validación
}

\author{
Adriana Coelho*1, Vítor Parola1', Miguel Escobar Bravo² y João Apóstolo
}

\begin{abstract}
'Instituto de Ciências Biomédicas Abel Salazar, Porto, Portugal; Unidade de Investigação em Ciências da Saúde: Enfermagem, Escola Superior de Enfermagem de Coimbra, Portugal; Portugal Centre for Evidence Based Practice: A JBI Centre of Excellence, Coimbra, Portugal. 'Universidad de Lleida, Facultad de Enfermería y Fisioterapia, Lleida, España. ${ }^{3}$ Unidade de Investigação em Ciências da Saúde: Enfermagem, Escola de Enfermagem de Coimbra, Portugal; Portugal Centre for A JBI Centre of Excellence, Coimbra, Portugal
\end{abstract}

Recibido el 1 de agosto de 2016

Aceptado el 17 de enero de 2017

PALABRAS CLAVE

Confort, cuidados paliativos, estudios de validación, cuidado terminal, cuidados paliativos al final de la vida.

\section{Resumen}

Objetivo: Traducir, adaptar y validar el Hospice Comfort Questionnaire para su utilización en el ámbito de los cuidados paliativos españoles.

Método: Han participado en este estudio un total de 67 pacientes ingresados en una Unidad de Cuidados Paliativos. El instrumento empleado ha sido el Hospice Comfort Questionnaire.

Se adoptó una metodología dirigida a la equivalencia semántica, idiomática y conceptual del contenido de los ítems y la equivalencia psicométrica, mediante la evaluación de la fiabilidad, la validez de contenido y de criterio.

Resultados: Respecto a la fiabilidad, se observaron comportamientos poco consistentes con el resto de la escala en siete ítems, que presentaran una correlación inferior a 0,20. Teniendo en cuenta los datos estadísticos y el análisis crítico de contenido de los ítems, se decidió eliminar solamente tres ítems. Una vez reducida la escala a 46 ítems, se midió la consistencia interna del total de la escala con un $\alpha$ de Cronbach de 0,89 y de cada grupo de ítems de cada estado del confort. El alfa de Cronbach del alivio fue de 0,72 , de la tranquilidad 0,73 , y de la trascendencia 0,75 . Se constató validez de criterio a través del coeficiente de correlación de Spearman, obteniendo una correlación de 0,805 entre el Hospice Comfort Questionnaire versión española y la Escala Visual Analógica de Confort.

Conclusiones: Los resultados muestran que la versión española del Hospice Comfort Questionnaire es un instrumento de evaluación de confort en cuidados paliativos con garantías psicométricas de calidad (buena fiabilidad y validez).

\footnotetext{
*Autor para correspondencia:

Adriana Coelho

Escola Superior de Enfermagem de Coimbra, Avenida Bissaya Barreto Apartado 7001, 3046-851 Coimbra, Portugal

Correo electrónico: adriananevescoelho@esenfc.pt.
} 
La adaptación al castellano del Hospice Comfort Questionnaire proporciona el acceso a una herramienta que permite a los profesionales elaborar un plan de cuidados lo más adecuado posible a las necesidades de confort de pacientes ingresados en Unidades de Cuidados Paliativos.

\section{KEYWORDS \\ Comfort, palliative \\ care, validation \\ studies, terminal \\ care, hospice care.}

\begin{abstract}
Objective: To translate, adapt and validate the Hospice Comfort Questionnaire for use in the context of palliative care Spanish.

Method: A total of 67 patients admitted in the Palliative Care Unit have participated in this study. The instrument used was the Hospice Comfort Questionnaire.

A methodology for the semantic, idiomatic and conceptual equivalence of items ' content and psychometric equivalence by assessing the reliability, validity and content approach was adopted.

Results: Regarding reliability, results inconsistent with the rest of the scale were observed in 7 items, with a correlation of less than 0.20 . Taking into account the statistical data and critical content analysis of the items, it was decided to eliminate only 3 items. Once the scale was reduced to 46 items, the internal consistency of the total scale was measured with a Cronbach's $\alpha$ of 0.89 and each group of items in each comfort state. Cronbach's alpha of relief was 0.72 ; ease 0.73 and transcendence 0.75 . Criteria validity was found through the Spearman correlation coefficient, obtaining a correlation of 0.805 between the Spanish version of the Hospice Comfort Questionnaire and the Visual Analog Scale of Comfort.

Conclusions: The results show that the Spanish version of the Hospice Comfort Questionnaire is an instrument for evaluating comfort in palliative care with psychometric quality assurance (good reliability and validity).

Spanish adaptation of Hospice Comfort Questionnaire provides access to a tool that allows professionals to develop more suitable care plan for comfort needs of patients admitted to palliative care units.
\end{abstract}

Coelho A, Parola V, Escobar Bravo M, Apóstolo J. Versión española del Hospice Comfort Questionnaire para evaluar el confort en pacientes en cuidados paliativos: adaptación transcultural y validación. Med Paliat. 2019;26(1):3-11.

\section{Introducción}

La práctica de los cuidados paliativos nace como respuesta al sufrimiento que el proceso de morir puede generar y procura integrar el alivio de los síntomas físicos, los aspectos psicológicos, espirituales y sociales, a fin de ofrecer el mayor confort y dignidad posible a los pacientes que se enfrentan a problemas asociados con enfermedades amenazantes para la vida ${ }^{1-4}$.

De hecho, Kolcaba ${ }^{5-7}$, en su teoría del confort, define confort como la experiencia de fortalecerse gracias a la satisfacción de las necesidades de alivio, tranquilidad y trascendencia. Según la autora, estas necesidades se desarrollan en cuatro contextos: físico, social, psicoespiritual y ambiental.

La conceptualización holística del confort es especialmente relevante para la atención en cuidados paliativos, ya que los pacientes que se sienten confortables, además de superan mejor los obstáculos, tienen una muerte más serena ${ }^{8,9}$.

A pesar de que el confort se define continuamente como un importante objetivo de los cuidados paliativos, en nuestro idioma y contexto no existen instrumentos para realizar su evaluación. En la actualidad se dispone de un amplio abanico de herramientas de evaluación que permiten realizar la valoración del paciente en situación avanzada de una manera unidimensional ${ }^{10-16}$. Probablemente, dicha varie- dad sea debida a que ningún instrumento se ha mostrado lo suficientemente útil como para ser aceptado de forma unánime ${ }^{17}$.

Sin embargo, la evaluación multidimensional constituye la esencia del proceso de atención integral en cuidados paliativos $^{17} y$, como tal, debe lograr información sobre el nivel de confort/disconfort de los enfermos. Disponer de esta información permitirá a los profesionales elaborar un plan de cuidados lo más adecuado posible a las necesidades de confort (físicas, sociales, psicoespirituales y ambientales) de cada paciente, definir la dirección para el cuidado interdisciplinario, realizar una mejor toma de decisiones terapéuticas e implementar intervenciones de confort ajustadas a las necesidades del paciente.

Se hace entonces imperativo disponer de un instrumento fiable y válido que evalúe el confort y facilite, además de una atención integral, la evaluación de los resultados de intervenciones de confort, congruentes con los objetivos de los cuidados paliativos.

Dada la falta de instrumentos para medir específicamente el confort total de los pacientes con enfermedad avanzada, Novak y cols. ${ }^{9}$, en base a la teoría del Confort de Kolcaba, han desarrollado el Hospice Comfort Questionnaire (HCQ).

El HCQ evalúa, específicamente, la satisfacción de los tres tipos de confort: alivio, tranquilidad y trascendencia. El alivio fue definido como el estado de un paciente que 
ha visto cumplida una necesidad específica. La tranquilidad fue definida como un estado de calma y satisfacción, es un estado duradero y positivo que es más que la ausencia de incomodidad. La trascendencia especifica el potencial del paciente, siendo definida como un estado en el cual un individuo está por encima de sus problemas o su dolor,18-20.

Los tres tipos de confort son evaluados en los cuatro contextos de la experiencia de confort: físico, psicoespiritual, social y ambiental. El contexto físico pertenece a las sensaciones corporales, como el dolor; el contexto psicoespiritual pertenece a la consciencia interna del yo, como la autoestima, el significado de la vida o la relación con un orden o estado superior; el contexto social se refiere a las relaciones interpersonales, familiares y sociales; finalmente, el contexto ambiental pertenece al entorno, las condiciones y las influencias externas como la luz, el ruido o la temperatura ${ }^{8,18-20}$ :

En síntesis, todos estos aspectos fueron cruzados en una tabla, constituyéndose así la estructura taxonómica del confort, que permite identificar e interpretar los varios componentes del confort para cada individuo ${ }^{8,18-20}$. Los ítems del Hospice Comfort Questionnaire, distribuidos de acuerdo con la estructura taxonómica del confort, pueden observarse en la Tabla I.

Hasta donde sabemos, el HCQ es el único instrumento creado específicamente para la evaluación del confort en el contexto de los cuidados paliativos.

Por lo anterior, se establece como objetivo de este estudio traducir, adaptar y validar el HCQ para su utilización en el ámbito de los cuidados paliativos españoles.

\section{Métodos}

\section{Participantes}

En el estudio han participado pacientes hospitalizados en la Unidad de Cuidados Paliativos (UCP) del Hospital de la Santa Creu de Vic.

Los criterios de inclusión fueron tener más de 18 años de edad, estar en condiciones de salud que le permitiesen rea- lizar el cuestionario (no presentar síntomas, en el momento de realización del cuestionario, que imposibilitaran la auto o heteroadministración del mismo, como por ejemplo somnolencia, dolor, dipnea, náuseas...), ausencia de deterioro cognitivo (mediante el test de Pfeiffer ${ }^{14}$ ), duración de estancia en la UCP igual o superior a 2 días (con el objetivo de proporcionar un periodo de tiempo que permitiera mejorar el control de síntomas, adaptación a cuestiones de orden práctico y a la propia hospitalización) y fluidez de la lengua castellana.

Como criterio de exclusión se consideró estar en situación de últimos días.

El tamaño muestral se calculó aceptando un nivel de confianza de 0,95 , una precisión de 8 y una desviación estándar de 27 (obtenida del estudio original ${ }^{9}$ ). Se estimó que el tamaño de la muestra sería de 44 participantes. En previsión de posibles pérdidas, teniendo en cuenta el riesgo de presentación de cuestionarios incompletos ${ }^{9}$, se aumentó en un $15 \%$ el tamaño muestral, administrándose finalmente el cuestionario a una muestra de 52 pacientes. Cabe señalar que este tamaño muestral es mayor que el del estudio original de validación, que se realizó con una muestra de 38 participantes.

\section{Materiales}

Las escalas que se incluyeron en este estudio fueron:

1. Hospice Comfort Questionnaire (HCQ) ${ }^{9}$.

El HCQ, desarrollado por Novak y cols., fue validado con 38 pacientes de dos UCP, y ha demostrado una alta fiabilidad al obtener valores del Alfa de Cronbach de 0,98. El HCQ está formado por 49 ítems que puntúan en una escala con formato tipo Likert, con anclajes que van de 1 ("totalmente en desacuerdo") a 6 ("totalmente de acuerdo"). La puntuación resultante puede variar entre 49 y 294, si se presenta la suma de las puntuaciones asignadas a cada ítem. Puntuaciones más altas indican un mayor nivel de confort. Los 49 ítems están formulados de forma positiva y negativa, para evitar sesgos ${ }^{8}$, y se plantean con la instrucción: "Las frases siguientes

Tabla I. Ítems del Hospice Comfort Questionnaire, distribuidos de acuerdo con la estructura taxonómica del confort.

\begin{tabular}{|c|c|c|c|c|}
\hline \multirow{2}{*}{$\begin{array}{l}\text { Contextos } \\
\text { (en los que ocurre } \\
\text { el confort) }\end{array}$} & \multicolumn{3}{|c|}{ Tipo de confort (estados) } & \multirow{2}{*}{ Número de ítems } \\
\hline & Alivio & Tranquilidad & Transcendencia & \\
\hline Físico & $\begin{array}{c}2^{*}, 5^{*}, 12^{*}, 14^{*}, 16 \\
19^{*}, 27^{*}, 31\end{array}$ & 1,36 & 29 & 11 \\
\hline Psicoespiritual & $24^{*}, 34^{*}, 46$ & $7,15,17^{*}, 35$ & $9,22^{*}, 41,45^{*}$ & 11 \\
\hline Ambiental & 3,49 & $11,18,25,32^{*}, 42$ & $21^{*}, 30^{*}, 33,38^{*}$ & 11 \\
\hline Social & $13^{*}, 26^{*}, 37,48^{*}$ & $\begin{array}{c}4,8,20,23,39^{*}, 40^{*} \\
43^{*}\end{array}$ & $6^{*}, 10,28,44,47$ & 16 \\
\hline Número de ítems & 17 & 18 & 14 & 49 \\
\hline
\end{tabular}


hacen referencia a su confort actual. Junto a cada pregunta encontrará seis números; rodee con un círculo el número que mejor refleje su estado. Responda a las preguntas según su confort en el momento actual".

Los ítems invertidos se encuentran discriminados en la Tabla I.

2. Escala Visual Analógica de Confort.

Se trata de una línea recta de $10 \mathrm{~cm}$ con un extremo marcado con "confort" y otro extremo que indica "disconfort". Se solicitó a cada participante que marcase en la línea el punto que mejor describía su nivel de confort en ese momento. La medición fue realizada en milímetros para proporcionar una medición precisa del confort total.

\section{Procedimiento}

El estudio se desarrolló en dos etapas: traducción y adaptación cultural del HCQ; y validación del HCQ en el idioma español.

Primera etapa: traducción y adaptación cultural del HCQ.

En esta etapa se tradujo el HCQ partiendo de su versión original en inglés. Se contactó con una de las autoras de la versión original (K. Kolcaba) y se le solicitó y obtuvo la autorización para su validación. El objetivo fue conseguir que el instrumento resultante mantuviera la equivalencia semántica, idiomática y conceptual con el cuestionario original.

Para ello se siguió la metodología de Beaton ${ }^{23}$ y Escobar ${ }^{24}$ para la traducción-retrotraducción de instrumentos, que incluyen los siguientes pasos:

- Traducción directa: se realizó una traducción conceptual del instrumento. En esta traducción participaron dos grupos de traductores bilingües independientes, cuya lengua materna era la española. Cada grupo estuvo compuesto por dos traductores. Uno de los grupos de traductores conocía los objetivos y los conceptos considerados en el cuestionario. El otro grupo de traductores no tenía conocimientos previos sobre el cuestionario y desconocía los objetivos del estudio.

- Síntesis de traducciones: los traductores y el equipo investigador discutieron las discrepancias entre las versiones traducidas hasta alcanzar el consenso, generando la primera versión del cuestionario en el idioma español.

- Traducción inversa (retro traducción): la versión de síntesis fue retro traducida al idioma original por dos grupos de traductores bilingües cuya lengua materna era la inglesa. Los grupos de traductores trabajaron de forma independiente, desconociendo la versión original del cuestionario, sin tener conocimientos previos sobre el tema y desconociendo los objetivos del estudio.

Se confirmó que la traducción no dio lugar a diferencias semánticas o conceptuales importantes entre el cuestionario original y la versión de síntesis obtenida en el paso anterior.

- Consolidación por un comité de expertos: en este paso se utilizaron las traducciones directas (paso 1), la ver- sión de síntesis (paso 2) y las retro traducciones (paso 3 ). Se constituyó un comité pluridisciplinario compuesto por el equipo investigador (experto en metodología: ME y JA, expertos en cuidados paliativos: AC y VP, expertos en confort: JA y AC), los traductores de la etapa preliminar, la autora del HCQ original (experta en confort) y una profesional sanitaria experta en cuidados paliativos. Este comité comparó y discutió estas versiones, generando la segunda versión del cuestionario en el idioma español.

- Pretest: el objetivo del pretest era determinar si los ítems que componen la versión experimental de la escala eran claros y estaban redactados sin ambigüedades, con un lenguaje ajustado al de la población diana. Entre diciembre de 2015 y enero de 2016, una muestra de 20 pacientes respondieron al cuestionario. Se pidió a estos pacientes que señalasen toda expresión o ítem ambiguo. No fueron señalados ítems ambiguos por los participantes. Así se obtuvo una versión traducida del HCQ adecuada en concepto y expresión a la versión original.

- Segunda etapa: validación del HCQ en el idioma español. Para esta etapa, previo consentimiento informado verbal y/o escrito del paciente y su aceptación en participar, así como previa autorización de la dirección del Hospital Universitario de la Santa Creu de Vic y de la valoración positiva del comité de ética de la Fundació d'Osona per a la Recerca i l'Educació Sanitàries (2015892 PR123), con el fin de garantizar un adecuado nivel en las propiedades psicométricas del instrumento, la versión final traducida del HCQ (Tabla II) fue cumplimentada por una muestra de 47 pacientes que cumplían los criterios de inclusión.

El cuestionario fue autoadministrado y, cuando hubo necesidad por cuestiones de debilidad y fragilidad, fue heteroadministrado por uno de los miembros del equipo investigador. La duración de la cumplimentación del cuestionario osciló entre 12 y 15 minutos. La recogida de los datos se realizó desde diciembre de 2015 hasta marzo de 2016.

Asimismo, se administró una escala EVA de Confort total a cada participante y un cuestionario relativo a las variables sociodemográficos. Los datos relativos a las variables clínicas se obtuvieron de la historia clínica de los pacientes. Estos datos pueden observarse en la Tabla III.

Los datos resultantes se introdujeron en una base de datos y se realizó un análisis de la validez (validez de contenido y validez de criterio), así como un análisis de la fiabilidad (consistencia interna) con el programa informático Statistical Package for Social Sciences (SPSS), versión 20.

\section{Análisis de datos}

En primer lugar, se calcularon estadísticos descriptivos y se estudió la consistencia interna de la escala a partir de las correlaciones ítem-total corregidas y el alfa total de la escala si se elimina el ítem (Tabla IV).

Según Streiner y Norman ${ }^{25}$, la correlación ítem-total de la escala inferiores a 0,20 deben ser eliminados. Sin embargo, 
Tabla II. Versión española del Cuestionario de confort en cuidados paliativos

Muchas gracias por ayudarnos en este estudio de enfermería en cuidados paliativos. Las frases siguientes hacen referencia a su confort actual. Junto a cada pregunta encontrará seis números; rodee con un círculo el número que mejor refleje su estado. Responda a las preguntas según su confort en el momento actual.

\begin{tabular}{|c|c|c|c|c|c|c|}
\hline & $\begin{array}{c}\text { Totalmente } \\
\text { en } \\
\text { desacuerdo }\end{array}$ & & & & & $\begin{array}{l}\text { Totalmente } \\
\text { de acuerdo }\end{array}$ \\
\hline 1. Mi cuerpo está relajado en este momento & 1 & 2 & 3 & 4 & 5 & 6 \\
\hline 2. Me cuesta respirar & 1 & 2 & 3 & 4 & 5 & 6 \\
\hline 3. Tengo suficiente intimidad & 1 & 2 & 3 & 4 & 5 & 6 \\
\hline $\begin{array}{l}\text { 4. Siempre encuentro a alguien en quien confiar } \\
\text { cuando lo necesito }\end{array}$ & 1 & 2 & 3 & 4 & 5 & 6 \\
\hline 5. Me preocupa mi familia & 1 & 2 & 3 & 4 & 5 & 6 \\
\hline 6. Mis creencias me aportan tranquilidad & 1 & 2 & 3 & 4 & 5 & 6 \\
\hline 7. Mis enfermeros/as me dan confianza & 1 & 2 & 3 & 4 & 5 & 6 \\
\hline 8. Mi vida vale la pena & 1 & 2 & 3 & 4 & 5 & 6 \\
\hline 9. Yo sé que soy querido/a & 1 & 2 & 3 & 4 & 5 & 6 \\
\hline 10. Este entorno es agradable & 1 & 2 & 3 & 4 & 5 & 6 \\
\hline 11. Tengo problemas para descansar & 1 & 2 & 3 & 4 & 5 & 6 \\
\hline 12. Nadie me entiende & 1 & 2 & 3 & 4 & 5 & 6 \\
\hline 13. Mi dolor es difícil de soportar & 1 & 2 & 3 & 4 & 5 & 6 \\
\hline 14. Me siento en paz & 1 & 2 & 3 & 4 & 5 & 6 \\
\hline 15. Duermo bien & 1 & 2 & 3 & 4 & 5 & 6 \\
\hline 16. Me siento culpable & 1 & 2 & 3 & 4 & 5 & 6 \\
\hline 17. Me gusta estar aquí & 1 & 2 & 3 & 4 & 5 & 6 \\
\hline 18. Siento náuseas & 1 & 2 & 3 & 4 & 5 & 6 \\
\hline 19. Puedo comunicarme con mis seres queridos & 1 & 2 & 3 & 4 & 5 & 6 \\
\hline $\begin{array}{l}\text { 20. Esta habitación hace que me sienta } \\
\text { asustado/a }\end{array}$ & 1 & 2 & 3 & 4 & 5 & 6 \\
\hline 21. Tengo miedo al futuro & 1 & 2 & 3 & 4 & 5 & 6 \\
\hline $\begin{array}{l}\text { 22. Tengo a una(s) persona(s) especial(es) que } \\
\text { hace }(\mathrm{n}) \text { que me sienta atendido/a }\end{array}$ & 1 & 2 & 3 & 4 & 5 & 6 \\
\hline $\begin{array}{l}\text { 23. He sufrido cambios que me hacen sentir } \\
\text { incómodo/a }\end{array}$ & 1 & 2 & 3 & 4 & 5 & 6 \\
\hline 24. Me gusta que mi habitación esté silenciosa & 1 & 2 & 3 & 4 & 5 & 6 \\
\hline $\begin{array}{l}\text { 25. Estoy satisfecho/a con mis relaciones } \\
\text { personales }\end{array}$ & 1 & 2 & 3 & 4 & 5 & 6 \\
\hline 26. Puedo sobreponerme a mi dolor & 1 & 2 & 3 & 4 & 5 & 6 \\
\hline 27. El ambiente de este lugar es triste & 1 & 2 & 3 & 4 & 5 & 6 \\
\hline 28. Me siento cómodo/a físicamente & 1 & 2 & 3 & 4 & 5 & 6 \\
\hline 29. La silla (cama) me provoca dolores & 1 & 2 & 3 & 4 & 5 & 6 \\
\hline 30. Estas vistas me animan & 1 & 2 & 3 & 4 & 5 & 6 \\
\hline 31. Pienso constantemente en mi malestar & 1 & 2 & 3 & 4 & 5 & 6 \\
\hline 32. Me siento seguro/a espiritualmente & 1 & 2 & 3 & 4 & 5 & 6 \\
\hline $\begin{array}{l}\text { 33. Me siento lo suficientemente bien como para } \\
\text { hacer cosas por mi cuenta }\end{array}$ & 1 & 2 & 3 & 4 & 5 & 6 \\
\hline $\begin{array}{l}\text { 34. Sé que mis amigos me recuerdan, porque me } \\
\text { escriben y/o me llaman }\end{array}$ & 1 & 2 & 3 & 4 & 5 & 6 \\
\hline 35. Aquí me siento fuera de lugar & 1 & 2 & 3 & 4 & 5 & 6 \\
\hline $\begin{array}{l}\text { 36. Necesito estar mejor informado/a sobre mi } \\
\text { enfermedad }\end{array}$ & 1 & 2 & 3 & 4 & 5 & 6 \\
\hline 37. Me siento desamparado/a & 1 & 2 & 3 & 4 & 5 & 6 \\
\hline 38. Dios me ayuda & 1 & 2 & 3 & 4 & 5 & 6 \\
\hline 39. Esta habitación huele a fresco & 1 & 2 & 3 & 4 & 5 & 6 \\
\hline 40. Me siento solo/a & 1 & 2 & 3 & 4 & 5 & 6 \\
\hline
\end{tabular}


Tabla II (Cont.). Versión española del Cuestionario de confort en cuidados paliativos

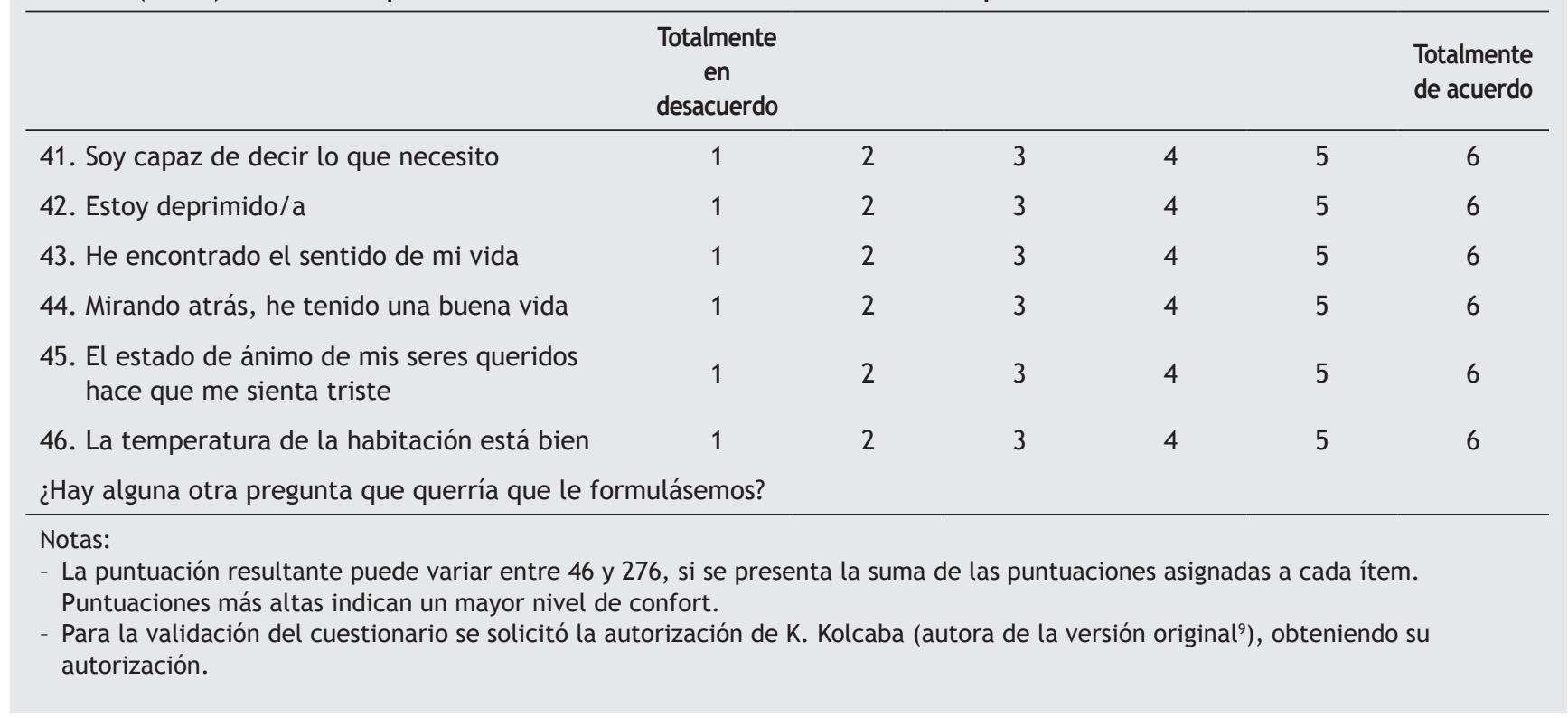

Tabla III. Características de los pacientes incluidos en la fase de validación

\begin{tabular}{lc}
\hline Características & Valores \\
\hline Edad (media)s & $73(47-89)$ \\
\hline Género & $62 \%(n=29)$ \\
Mujeres & $38 \%(n=18)$ \\
Hombres & \\
\hline Diagnóstico & $79 \%(n=37)$ \\
Oncológico & $21 \%(n=10)$ \\
No oncológico & \\
\hline Estado civil & $79 \%(n=37)$ \\
Casados & $17 \%(n=8)$ \\
Viudos & $4 \%(n=2)$ \\
Divorciados & \\
\hline Nivel de escolaridad & $28 \%(n=13)$ \\
Sin estudios & $38 \%(n=18)$ \\
Educación primaria & $23 \%(n=11)$ \\
Educación secundaria & $11 \%(n=5)$ \\
Educación superior & \\
\hline
\end{tabular}

el equipo investigador no tuvo solamente en cuenta los datos estadísticos, sino también el análisis crítico del contenido de los ítems en que se observaron comportamientos poco consistentes con el resto de la escala con vista a determinar su eliminación o no.

Con los ítems restantes, se estimó la consistencia interna del HCQ-versión ES mediante el coeficiente alfa de Cronbach. Se considera que existe una buena consistencia interna cuando el valor de alfa es superior a 0,7 y se considera una exce- lente consistencia cuando el valor es superior a 0,926,27. Sin embargo, una puntuación demasiado alta (superior a 0,9) puede hacer suponer que los ítems del instrumento miden un aspecto demasiado restrictivo del concepto ${ }^{24}$.

Posteriormente, se estimó la validez de criterio (validez concominante) del HCQ-versión ES mediante la medición del grado de correlación entre los resultados del HCQ-versión ES y de la EVA de Confort aplicadas al mismo tiempo y en los mismos sujetos.

\section{Resultados}

\section{Características de los pacientes}

Han participado en el estudio un total de 72 pacientes ingresados en la UCP, 20 en la primera etapa y 52 en la segunda etapa. Cinco participantes de la segunda etapa fueron excluidos del estudio por encontrarse el cuestionario incompleto y por imposibilidad de volverlo a administrar por debilidad clínica.

Los 47 participantes incluidos en la segunda etapa del estudio presentaban una edad media de 73 años, de los que un $62 \%$ eran mujeres, y el $79 \%$ presentaba una enfermedad oncológica. Las características de la muestra pueden observarse en la Tabla III.

\section{Propiedades psicométricas del instrumento}

La revisión se realizó por traductores bilingües y profesionales sanitarios con altos conocimientos en inglés, expertos en el tema, y una de las autoras del instrumento original que compararon la versión original con la versión obtenida. Este proceso proporciona garantías sobre la validez de contenido de la nueva escala. 
Tabla IV. Estadísticos descriptivos de los ítems de la escala.

\begin{tabular}{|c|c|c|c|c|}
\hline Ítem & $M$ & DT & r total elemento corregida & $\alpha$ si se elimina el elemento \\
\hline 1 & 4,89 & 1,005 & 0,453 & 0,879 \\
\hline 2 & 4,26 & 1,621 & 0,219 & 0,882 \\
\hline 3 & 4,53 & 1,365 & 0,184 & 0,882 \\
\hline 4 & 5,55 & 0,544 & 0,228 & 0,881 \\
\hline 5 & 4,36 & 1,905 & 0,069 & 0,885 \\
\hline 6 & 2,34 & 1,845 & 0,266 & 0,881 \\
\hline 7 & 4,36 & 1,405 & 0,388 & 0,879 \\
\hline 8 & 5,36 & 0,705 & 0,226 & 0,881 \\
\hline 9 & 5,04 & 1,233 & 0,492 & 0,878 \\
\hline 10 & 5,55 & 0,544 & 0,240 & 0,881 \\
\hline 11 & 5,30 & 0,976 & 0,445 & 0,879 \\
\hline 12 & 3,91 & 1,863 & 0,638 & 0,874 \\
\hline 13 & 4,32 & 1,795 & 0,465 & 0,877 \\
\hline 14 & 3,30 & 1,667 & 0,406 & 0,879 \\
\hline 15 & 5,19 & 1,116 & 0,353 & 0,880 \\
\hline 16 & 4,70 & 1,382 & 0,576 & 0,876 \\
\hline 17 & 4,87 & 1,715 & 0,292 & 0,881 \\
\hline 18 & 4,30 & 1,743 & 0,226 & 0,882 \\
\hline 19 & 5,04 & 1,367 & 0,072 & 0,883 \\
\hline 20 & 5,51 & 0,748 & 0,093 & 0,882 \\
\hline 21 & 5,06 & 1,436 & 0,528 & 0,877 \\
\hline 22 & 3,72 & 1,942 & 0,426 & 0,878 \\
\hline 23 & 5,32 & 0,726 & 0,356 & 0,880 \\
\hline 24 & 2,66 & 1,478 & 0,242 & 0,881 \\
\hline 25 & 5,06 & 1,150 & $-0,036$ & 0,884 \\
\hline 26 & 2,91 & 1,265 & $-0,072$ & 0,885 \\
\hline 27 & 2,06 & 1,275 & 0,042 & 0,884 \\
\hline 28 & 5,04 & 1,197 & 0,242 & 0,881 \\
\hline 29 & 4,72 & 0,800 & 0,383 & 0,880 \\
\hline 30 & 3,96 & 1,615 & 0,622 & 0,875 \\
\hline 31 & 4,30 & 1,140 & 0,617 & 0,876 \\
\hline 32 & 3,94 & 1,660 & 0,341 & 0,880 \\
\hline 33 & 5,15 & 1,215 & 0,525 & 0,877 \\
\hline 34 & 3,43 & 1,815 & 0,322 & 0,880 \\
\hline 35 & 5,02 & 1,093 & 0,320 & 0,880 \\
\hline 36 & 3,79 & 1,429 & 0,455 & 0,878 \\
\hline 37 & 5,32 & 0,629 & 0,511 & 0,879 \\
\hline 38 & 3,83 & 1,698 & 0,409 & 0,878 \\
\hline 39 & 3,11 & 1,735 & 0,217 & 0,882 \\
\hline 40 & 5,04 & 1,334 & 0,688 & 0,875 \\
\hline 41 & 4,32 & 1,656 & 0,426 & 0,878 \\
\hline 42 & 5,11 & 0,759 & 0,320 & 0,880 \\
\hline 43 & 4,79 & 1,614 & 0,577 & 0,876 \\
\hline 44 & 5,40 & 0,771 & 0,377 & 0,880 \\
\hline 45 & 3,98 & 1,648 & 0,520 & 0,876 \\
\hline 46 & 3,96 & 1,532 & 0,368 & 0,879 \\
\hline 47 & 4,91 & 1,231 & 0,373 & 0,879 \\
\hline 48 & 3,40 & 1,849 & 0,435 & 0,878 \\
\hline 49 & 5,09 & 1,100 & 0,336 & 0,880 \\
\hline
\end{tabular}


Respecto a los resultados referentes a la fiabilidad, se observaron comportamientos poco consistentes con el resto de la escala en siete ítems: 3, 5, 19, 20, 25, 26 y 27, que presentaran una correlación inferior a 0,20.

Teniendo en cuenta los datos estadísticos y el análisis crítico de contenido de los ítems, se decidió eliminar solamente los ítems 5, 25 y 26. Se decidió mantener los ítems 3, 19, 20 y 27 por considerar su contenido muy relevante para la evaluación del confort y, además, porque la eliminación de estos ítems no generaba un aumento del Alpha de Cronbach (alfa de la escala con los 49 ítems $=0,88$ ).

Se decidió eliminar el ítem 5 ("Me siento hinchado/a") por generar ambigüedad entre los participantes, ya que el término "hinchado" tuvo doble interpretación (edema y/o estreñimiento). Lamentablemente esta ambigüedad no fue detectada en el pretest. Se decidió eliminar los ítems 25 ("Me gusta que mi habitación esté silenciosa") y 26 ("Me gustaría que el médico me visitase más a menudo") por, además de ser los únicos ítems que presentaban una correlación negativa con el resto de la escala, se consideró su contenido/interpretación ambiguo/a: la necesidad de silencio no necesariamente representa confort, ya que puede representar soledad ${ }^{28}$, y el deseo de aumento de periodicidad de visitas por parte del médico no necesariamente significa disconfort. Muchas veces el médico es el portador de "malas noticias" y pacientes que estén en negación no desean un aumento de visitas por parte del médico.

Una vez reducida la escala a 46 ítems, se realizó el análisis de la consistencia interna del total de la escala $(0,89)$ y a cada grupo de ítems de cada estado del confort. El alfa de Cronbach del alivio fue de 0,72 , de la tranquilidad 0,73 y de la trascendencia 0,75 . Todas las estimaciones indicaron una buena consistencia interna del HCQ-versión ES.

Posteriormente, para determinar la validez de criterio, se calculó el grado de correlación entre el HCQ-versión ES y la EVA a través del coeficiente de correlación de Spearman, obteniendo una correlación elevada $(0,805)$, que demuestra la validez de la escala.

Finalmente se calcularon los estadísticos descriptivos para la puntuación global de confort, que reveló una media de $204,7(D E=26,2)$, con puntuaciones que oscilaron entre 121 y 257.

Hay que destacar que no fueran encontradas diferencias estadísticamente significativas en el nivel de confort total de hombres y mujeres $(p=0,192)$, de los pacientes con enfermedad oncológica y no oncológica $(p=0,535)$, de los pacientes casados, divorciados y viudos $(p=0,191)$, de los pacientes con edad inferior o igual a 65 años y superior a 65 años ( $p=0,832)$, ni de los pacientes sin estudios, con educación primaria, secundaria y superior $(p=0,423)$.

\section{Discusión}

Puesto que el confort es un importante objetivo de los cuidados paliativos, la evaluación del estado de confort y la detección de disconfort es de suma importancia.

El presente estudio aporta una herramienta de diagnóstico y de evaluación de los resultados de la implementación de intervenciones de confort que era inexistente hasta al momento.
El estudio de la validez concomitante reveló una correlación elevada entre el HCQ-versión ES y la EVA. Este dato confirma la validez de la escala, pero también suscita algunas cuestiones respecto a la necesidad de implementar un cuestionario tan extenso como el HCQ-versión ES. Así, es importante subrayar que el HCQ-versión ES, al contrario de la EVA, permite a los profesionales elaborar un plan de cuidados lo más adecuado posible a las necesidades específicas de confort físicas, sociales, psicoespirituales y ambientales de cada paciente.

El HCQ-versión ES, además de evaluar y ayudar a la detección precoz de necesidades de confort, sirve como guía terapéutica y favorece la comunicación entre profesionales y pacientes. Es decir, si bien la aplicación del HCQ-versión ES puede resultar demasiado extensa para pacientes en situación vulnerable, como los de Cuidados Paliativos, en los que resulta complicado pasar diferentes escalas de valoración para su adecuada atención dependiendo de su estado clínico, también es importante señalar que la aplicación del cuestionario a la muestra del estudio no ha supuesto un esfuerzo físico o emocional inapropiado para los participantes. Además, tal y como recomiendan los autores del instrumento original ${ }^{9}$ y los autores de otros instrumentos con vista a su aplicación en el contexto paliativo ${ }^{29}$, en situación de imposibilidad de que el cuestionario fuera autoadministrado se optó por la heteroadministración.

Cuando el cuestionario fue heteroadministrado, su administración constituyó un momento de elevada importancia para el participante y el investigador, dado que todos los participantes han manifestado interés en hablar de forma más profunda respecto a cada ítem, añadiendo información que superaba los objetivos de la presente investigación pero con elevado interés en el ámbito del estudio del confort en cuidados paliativos, y en el ámbito de la prestación de cuidados con vista a la satisfacción de las necesidades de confort del enfermo.

A pesar de que la versión original del HCQ esté destinada a ser autoadministrada y heteroadministrada', se sugiere que el instrumento sea heteroadministrado y se estima que la actitud con la que el profesional sanitario se acerque al paciente para administrar el HCQ-versión ES pueda influir en el verdadero sentido del HCQ-versión ES.

Entre las limitaciones de este estudio destacamos el reducido tamaño de la muestra que se justifica por las características de vulnerabilidad y fragilidad de los participantes, y porque el estudio fue realizado en una misma ubicación. Esta limitación imposibilitó la estimación de la validez de constructo $^{25}$.

La progresiva debilidad de los participantes y el respeto por la dignidad humana que siempre está por encima de los intereses de la investigación llevó al equipo a no estudiar la estabilidad del instrumento (Test-retest).

De cara a posteriores investigaciones, dadas las dificultades y limitaciones a nivel del tamaño de la muestra en estudios con pacientes con enfermedad avanzada ${ }^{30}$, sería adecuada la realización de un estudio multicéntrico.

Asimismo, el HCQ-versión ES se ha administrado a pacientes oncológicos y no oncológicos avanzados hospitalizados en una UCP, por lo que su posible aplicación a pacientes con enfermedades avanzadas hospitalizados en otras unidades que también reciban a pacientes con necesidad de atención paliativa debería verificarse en otros estudios. 
Finalmente, los niveles de funcionalidad de los participantes, además de no haber sido definidos como criterio de inclusión del estudio, tampoco fueron tenidos en cuenta en la recogida de datos clínicos. Es recomendable que en futuras investigaciones los niveles de funcionalidad de los participantes, y otras variables clínicas, sean tenidos en cuenta para posibilitar la estimación de la relación entre los niveles de confort presentados por los participantes y su nivel de funcionalidad.

En este sentido, sería interesante la validación de este instrumento en un estudio multicéntrico donde estuvieran representados pacientes de cuidados paliativos de diferentes centros y diferentes complejidades clínicas.

No obstante a lo anterior, el HCQ-versión ES se ha revelado como un instrumento fiable y válido para evaluar el confort de los pacientes ingresados en UCP, con lo cual ya se puede disponer de una herramienta complementaria de trabajo clínico para la valoración del grado de confort de estos pacientes.

\section{Agradecimientos}

A todos los pacientes que han participado en este estudio; a la Unidad de Investigación en Ciencias de la Salud: Enfermería, acogida por la Escuela Superior de Enfermería de Coímbra (ESEnfC), Portugal; al Hospital Universitario de la Santa Creu de Vic. Catalunya.

\section{Conflicto de intereses}

Los autores declaran no tener ningún conflicto de intereses.

\section{Bibliografía}

1. Worldwide Palliative Care Alliance. Global Atlas of Palliative Care at the End of Life. London; 2014.

2. Bayés R. Morir en paz: evaluación de los factores implicados. Med Clin (Barc). 2004;122(14):539-41.

3. Twycross R. Medicina paliativa: filosofía y consideraciones éticas. Acta Bioeth. 2000;6(1):29-46.

4. Guía de Cuidados Paliativos [Internet]. Sociedad española de Cuidados Paliativos; 2002.

5. Kolcaba K. Evolution of the mid range theory of comfort for outcomes research. Nurs Outlook. 2001;49(2):86-92.

6. Kolcaba KY. Comfort as Process and Product, Merged in HolisticNursing Art. J Holist Nurs. 1995;13(2):117-31.

7. Vendlinski S, Kolcaba K. Comfort care: A framework for hospice nursing. J Hosp Palliat Care. 1997;14(6):271-6.

8. Kolcaba K. Comfort Theory and Practice: A Vision for Holistic Health Care and Research. New York: Springer Publishing Company; 2003.

9. Novak B, Kolcaba K, Steiner R, Dowd T. Measuring comfort in caregivers and patients during late end-of-life care. Am J Hosp Palliat Care. 2001;18(3):170-80.

10. Badia X, Muriel C, Gracia A, Núñez-Olarte JM, Perulero N, Gálvez R, et al. Validación española del cuestionario Brief Pain Inventory en pacientes con dolor de causa neoplásica. Med Clin. 2003;120(2):52-9.
11. Kaasa T, Loomis J, Gillis K, Bruera E, Hanson J. The Edmonton Functional Assessment Tool: Preliminary development and evaluation for use in palliative care. J Pain Symptom Manage. 1997;13(1):10-9.

12. Reig-ferrer A, Ferrer-cascales R, Dolores M. Evaluación del bienestar espiritual en pacientes en cuidados. Med Paliativa. 2016;22(2):60-8.

13. Pargament KI, Koenig HG, Perez LM. The many methods of religious coping: development and initial validation of the RCOPE. J Clin Psychol. 2000;56(4):519-43.

14. Martínez de la Iglesia J, Dueñas Herrero R, Onís Vilches $M$, Taberné C, Colomer C, Luque R. Adaptación y validación al castellano del cuestionario de Pfeiffer (SPMSQ) para detectar la existencia de deterioro cognitivo en personas mayores e 65 años. Med Clin (Barc). 2001;117(4):129-34.

15. Centeno Cortés C. Algunos instrumentos de evaluación utilizados en cuidados paliativos (I) El cuestionario de evaluación de síntomas de Edmontón (ESAS). Med paliativa. 2004;11(4):239.

16. Lobo A, Saz P, Marcos G, Día Sahun JL, de la Cámara C, Ventura T, et al. Revalidación y normalización del Mini-Examen Cognoscitivo (primera versión en castellano del Mini-Mental Status Examination) en la población general geriátrica. Med Clin (Barc). 1999;112(20):767-74.

17. Garzón Rodríguez C, Martínez Losada E, Julia Torras J, González Barboteo J, Maté Méndez J, Ochoa Arnedo C, et al. Herramientas para la evaluación multidimensional de uso para médicos de Cuidados Paliativos: Proyecto ICO-tool kit. Med Paliativa. 2010;17(6):348-59.

18. Kolcaba K. A taxonomic structure for the concept comfort. Imagen J Nurs Sch. 1991;23(4):237-40.

19. Kolcaba KY. Holistic comfort: Operationalizing the construct as a nurse- sensitive outcome. Adv Nurs Sci. 1992;15(1):1-10.

20. Kolcaba K. A theory of holistic comfort for nursing. J Adv Nurs. 1994;19(6):1178-84.

21. Apóstolo JLA. O imaginário conduzido no conforto de doentes em contexto psiquiátrico. Universidade do Porto; 2007.

22. Matos M. O Conforto da Família em Cuidados Paliativos - Tradução e Validação para a População Portuguesa do Holistic Comfort Questionnaire (Family). Universidade Católica Portuguesa; 2012.

23. Beaton DE, Bombardier C, Guillemin F, Ferraz MB. Guidelines for the process of cross-cultural adaptation of self-report measures. Spine (Phila Pa 1976). 2000;25(24):3186-91.

24. Escobar Bravo MA. Adaptación transcultural de instrumentos de medida relacionados con la salud. Enfermería Clínica. 2004;14(2):102-6.

25. Streiner D, Norman G. Health measurement scales: a practical guide to the development and use. $4 .^{\text {a }}$ ed. New York: Oxford University Press; 2008.

26. Arribas M. Diseño y validación de cuestionarios. Matronas Profesión. 2004;5(17):23-9.

27. Carvajal A, Centeno C, Watson R, Martínez M, Sanz-Rubiales A. ¿Cómo validar un instrumento de medida de la salud? An Sist Sanit Navar. 2011;34(1):63-72.

28. Coelho A, Parola V, Escobar-Bravo M, Apóstolo J. Comfort experience in palliative care: a phenomenological study. BMC Palliat Care. 2016;15(1):71.

29. Pinto S, Caldeira S, Martins J, Kolcaba K. Cultural adaptation and validation of the Portuguese End of Life Spiritual Comfort Questionnaire in Palliative Care patients. Porto Biomed J. PBJAssociação Porto Biomedical/Porto Biomedical Society; 2016;6-11.

30. Requena López A, Moreno Mateo R, Rodeles del Pozo R, Torrubia Atienza MP, Nabal Vicuña M, Domínguez Barcelona LM. Validación de un modelo pronóstico de supervivencia basado en parámetros biológicos para pacientes con cáncer terminal atendidos en ámbito domiciliario. Med Paliativa. 2013;20(1):3-9. 